\title{
Reciclaje para el desarrollo de cultura ambiental en estudiantes del CEBE
}

\author{
Vilma Marisol Paredes Vega \\ vparedesv@ucvvirtual.edu.pe \\ https://orcid.org/0000-0002-2895-0169
}

Norma Amparo Chacón Enríquez CHENRIQUEZV@ @ ucvvirtual.edu.pe https://orcid.org/0000-0001-6993-1530

Groberti Alfredo Medina Corcuera grome@ucvvirtual.edu.pe https://orcid.org/0000-0003-4035-157X

Universidad César Vallejo Chimbote- Perú

\section{RESUMEN}

La investigación desarrolló el objetivo implementar estrategias de reciclaje para el desarrollo la cultura ambiental de los niños, docentes y padres de familia del CEBE Fe y Alegría $\mathrm{N}^{\circ} 42$, 2021, el enfoque empleado fue cuantitativo, el tipo de investigación un diseño pre-experimental; la población fue de 170 estudiantes, la muestra 17 niños y niñas con habilidades diferentes del nivel primaria, la técnica la encuesta, los instrumentos pre y post test. La hipótesis fue: La Implementación de estrategias de reciclaje si desarrollará significativamente la cultura ambiental en estudiantes y padres de familia. Al analizar los resultados el nivel logro destacado del pre test no se tiene registro y en el post test se registró el nivel logro destacado en 14 estudiantes que conforman el 66.7\%, asimismo, además se tiene la prueba $\mathrm{T}$ de Student, se ha logrado obtener un valor T observado de 11,040 considerado como superior al valor T tabular 1,725 (20 grados de libertad), el valor de la significancia obtenida se tiene un valor de 0.000 , situado por debajo del 0.05 . por tal se concluyó que los niños han logrado el nivel.

Palabras clave: plan de gestión; gestión ambiental; actitudes ambientales. 


\title{
Recycling for the development of environmental culture in CEBE students
}

\begin{abstract}
The investigation developed the objective to implement strategies of recycling, to develop the culture of the environment of the children, teachers, and family parents of CEBE Faith and Joy, no. 42, 2021. The focus used was a quantitative study, i.e., a type of preexperimental design study: the members were made up of 170 students, 17 professors, and 17 boys and girls with different backgrounds from primary grades, the technique of inquiry, the instruments pre- and post-test. The hypothesis was as follows: the implementation of strategies of recycling will develop significantly the culture of the environment among the students, the family parents. The analysis of the results on the level attained from the pre-test and the results of the post-test showed a gain of $66>7 \%$. Also, there was the test $\mathrm{T}$ of the student, which showed a value $\mathrm{T}$ observed of the 11,140 considered to be superior to the value T tabulated 1,725 (20 grades of freedom). The value of the significance obtained showed a value of 0.00 , situated below 0.05 . From this it was concluded that the children had gained the level.
\end{abstract}

Key words: plan of management; plan of environment; and environmental attitudes.

Artículo recibido: 02 noviembre. 2021 Aceptado para publicación: 28 noviembre 2021

Correspondencia: vilpa0400@gmail.com Conflictos de Interés: Ninguna que declarar 


\section{INTRODUCCIÓN}

La contaminación es un problema que cada día profundiza sus efectos como consecuencia directa de la actividad humana tanto a gran escala en las inmensas fábricas, explotaciones mineras, refinerías y otros, así como lo más pequeños espacios donde nos desenvolvemos cotidianamente como los centros de abastos, colegios y hogares.

A nivel mundial, la generación en grandes cantidades y el mal aprovechamiento de los residuos sólidos, es un problema alarmante; según el Banco Mundial (2018), los países desarrollados representan aprox. el $16 \%$ de la ciudadanía, pero originan cantidades superiores a la tercera parte (34\%) de los residuos a nivel mundial. Así también el Pacífico y Asia oriental, cerca de la cuarta parte (23\%) del total. Y se calcula que dentro de 30 años la generación y acumulación de desechos sólidos en el mundo se incrementará en un $70 \%$ con respecto a los niveles actuales; siendo el plástico el que representa cifras alarmantes.

En el Perú, la situación no es ajena; de acuerdo al sistema de información ambiental local, solo en la capital se produce cantidades supriores a ocho mil toneladas diarias de residuos, cifra que representa el $45 \%$ de todo el Perú. Casi la totalidad de los distritos de Lima y el Callao han implementado sistemas de acopio de desechos sólidos, Sin embargo, la mayor parte de los desechos recolectados son orgánicos y pueden ser reutilizados para compost. Otro producto es el plástico, papel y vidrio que también pueden ser reaprovechados, aunque solo el $4 \%$ diario van para ese fin o al reciclaje formal.

Frente a ello, el manejo apropiado de residuos sólidos principalmente en espacios urbanos ha crecido importantemente en los últimos años, en busca de mantener limpias las ciudades y en armonía ambiental, promovida de la legislatura peruana mediante de programas, en el marco de la normatividad internacional, adoptando un enfoque ambientalista. Aun así, dicho tema sigue siendo inadecuado generando impacto dañino para el ambiente, así como la salud de quienes se ocupan de esta actividad y por ende a la economía del país, puesto que se tendría una problemática de bienestar público que atender.

En la búsqueda de revertir este panorama, si bien es cierto ha habido avances desde el ámbito municipal, a la fecha son muchas las municipalidades a nivel nacional que están poniendo en marcha programas para un tratamiento pertinente de desechos sólidos, 
fundamentalmente los residentes asociados al aseo público, como el "Programa de Segregación en la Fuente y Recolección Selectiva de Residuos Sólidos” en adelante PSFRSRS; creado en el 2011 por el Ministerio del Ambiente.

Pero si el propósito es el manejo idóneo de los residuos es necesario un PSFRSRS para las II.EE, en concordancia con el Ministerio de Educación que también es considerado como un actor del PSFRSRS y es concebido en Rentería y Zevallos (2014) como una entidad del Estado cuyo fin es garantizar derechos, asegurando un servicio educativo de calidad y mediante las UGEL se coordina con las Instituciones Educativas la preparación a los docentes, facilitándoles materiales relacionados a temas la preservación del ambiente así como la segregación de residuos, con el fin de generar una cultura y hábito del reciclaje.

En función a todo, se plantea problema de la siguiente manera: ¿De qué manera el reciclaje permitirá el avance de la cultura ambiental en estudiantes y padres de familia del CEBE Fe Y Alegría N², 2021?; dicho trabajo de investigación se justifica en el sentido práctico, en tanto fortalecer las conductas positivas relacionadas con cuidar el entorno resulta una relevante alternativa de generación de conciencia ambiental y una manera de valorar en nivel de cultura ambiental que poseen los estudiantes; sin embargo, se requiere tener conocimiento avanzado acerca de las conductas que los estudiantes posee respecto a su entorno, este saber posibilitará que se diseñen programas pertinentes que fomenten en ellos los comportamientos deseados y se formen adecuadamente.

Se justifica también por que la educación ambiental y el reciclaje, son procedimientos continuos que posibilitan el progreso y el proyectarse al futuro por parte de la colectividad, de igual manera se presenta como técnica relevante para generar cambios en la cultura en los estudiantes, exteriorizado en valores y cualidades que beneficien a la construcción de una sociedad más equitativa y armoniosa en la que los factores políticos, sociales, ambientales y económicos aseguren un desarrollo sustentable para la población. Se justifica el trabajo en el sentido metodológico porque, se elaborará un instrumento de recojo de información el que se le hará una prueba de confiabilidad y validación por expertos en el tema, documento que servirá para próximas investigaciones.

Asimismo, se planteó los objetivos de investigación, general: Implementar estrategias de reciclaje para el desarrollo la cultura ambiental de los niños, docentes y padres de familia 
del CEBE Fe y Alegría N 42, 2021. Paralelamente, se planteó los objetivos específicos: Comparar los resultados del equilibrio ecológico antes y después de aplicar las estrategias de reciclaje en niños, docentes y padres de familia del CEBE Fe y Alegría $\mathrm{N}^{\circ}$ 42, 2021. Comparar los resultados de los valores ambientales antes y después de aplicar las estrategias de reciclaje en niños, docentes y padres de familia del CEBE Fe y Alegría $\mathrm{N}^{\circ}$ 42, 2021. Comparar los resultados del consumo responsable antes y después de aplicar las estrategias de reciclaje en niños, docentes y padres de familia del CEBE Fe y Alegría $\mathrm{N}^{\circ}$ 42, 2021. Comparar los resultados de la calidad de vida antes y después de aplicar las estrategias de reciclaje en niños, docentes y padres de familia del CEBE Fe y Alegría $\mathrm{N}^{\circ}$ 42, 2021.

Y para responder a la interrogante investigativa se plantearon las hipótesis de investigación (Hi): La Implementación de estrategias de reciclaje si desarrollará significativamente la cultura ambiental en estudiantes y padres de familia del CEBE Fe Y Alegría $\mathrm{N}^{\circ} 42$, 2021. Y una hipótesis nula, (Ho): la Implementación estrategias de reciclaje no desarrolla la cultura ambiental en estudiantes y padres de familia del CEBE Fe Y Alegría N42, 2021.

\section{ESTRATEGIAS METODOLÓGICAS O MATERIALES Y MÉTODOS}

La investigación, presenta un esquema Pre-experimental y de corte transversal el cual es interpretado por Hernández, Fernández y Baptista (2014) de la siguiente manera:

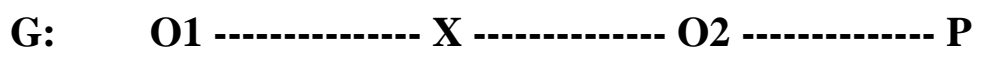

\section{Donde:}

G: Es la agrupación de integrantes

O1: Medición previa al experimento de la variable dependiente

$\mathrm{X}$ : Es la aplicación del experimento

O2: Medición posterior al experimento de la variable dependiente

P : Propuesta 


\section{RESULTADOS Y DISCUSIÓN}

\subsection{Resultados}

\section{Análisis descriptivo}

Tabla 1 Implementar estrategias de reciclaje para el desarrollo la cultura ambiental de los niños, docentes y padres de familia del CEBE Fe y Alegría $N^{\circ}$ 42, 2020 - 2021.

\begin{tabular}{lccccccc}
\hline \multicolumn{1}{c}{ Niveles } & \multicolumn{2}{c}{ Pre Test } & \multicolumn{2}{c}{ Post Test } & \multicolumn{2}{c}{ Diferencia } \\
& $\mathbf{f i}$ & $\mathbf{\%}$ & $\mathbf{f i}$ & $\mathbf{\%}$ & $\mathbf{f i}$ & $\boldsymbol{\%}$ \\
\hline Inicio & 6 & $28.6 \%$ & 0 & $0.0 \%$ & 6 & $28.6 \%$ \\
Proceso & 11 & $52.4 \%$ & 1 & $4.8 \%$ & 10 & $47.6 \%$ \\
Logro Esperado & 4 & $19.0 \%$ & 6 & $28.6 \%$ & 2 & $9.5 \%$ \\
Logro Destacado & 0 & $0.0 \%$ & 14 & $66.7 \%$ & 14 & $66.7 \%$ \\
Total & 21 & $100.0 \%$ & 21 & $100.0 \%$ & & \\
\hline
\end{tabular}

Fuente: Base de datos del pre y post test.

Figura 1. Frecuencia de las estrategias de reciclaje para el desarrollo la cultura ambiental de los niños, docentes y padres de familia del CEBE Fe y Alegría $N^{\circ}$ 42, $2020-2021$

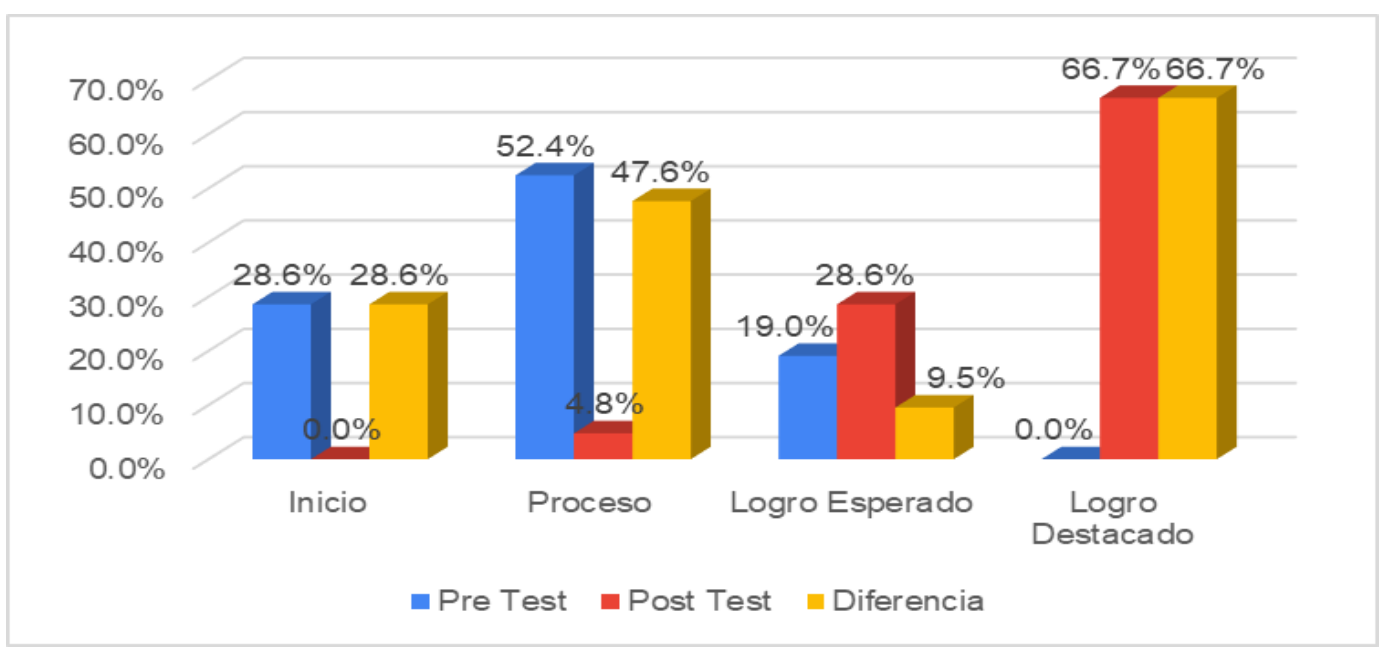

Fuente: Tabla 1

\section{Interpretación:}

A partir de los resultados de la comparación realizada en el pre y post test se tiene que en el nivel de inicio en el pre test se ha evidencia a 6 estudiantes que representan el $28.6 \%$, luego en el post test se ha registrado a ningún estudiante, logrando disminuir en $28.6 \%$; de ahí se tiene que en el nivel proceso en el pre test se ha evidenciado que 11 estudiantes que conforman el 52.4\% y en el post test se ha mostrado a 1 estudiante que conforma el $4.8 \%$, logrando una mejora del $47.6 \%$. Luego en el nivel logro esperado se tiene en el pre test a 4 estudiantes que conforman el 19.0\%, luego en el post test se tiene que 6 estudiantes que conforman el $28.6 \%$, haciendo una diferencia del 9.5\%. Finalmente, en el nivel logro 
destacado en el pre test no se tiene registro y en el post test se ha registrado a 14 estudiantes que conforman el 66.7\%. haciendo una diferencia del 66.7\%, demostrando la efectividad que presento las estrategias de reciclaje.

Tabla 2. Comparar los resultados del equilibrio ecológico antes y después de aplicar las estrategias de reciclaje en niños, docentes y padres de familia del CEBE Fe y Alegría $N^{\circ}$ $42,2020-2021$.

\begin{tabular}{lcccccc}
\hline \multirow{2}{*}{ Niveles } & \multicolumn{2}{c}{ Pre Test } & \multicolumn{2}{c}{ Post Test } & \multicolumn{2}{c}{ Diferencia } \\
& $\mathbf{f i}$ & $\mathbf{\%}$ & $\mathbf{f i}$ & $\mathbf{\%}$ & $\mathbf{f i}$ & $\mathbf{\%}$ \\
\hline Inicio & 15 & $71.4 \%$ & 1 & $4.8 \%$ & 14 & $66.7 \%$ \\
Proceso & 4 & $19.0 \%$ & 2 & $9.5 \%$ & 2 & $9.5 \%$ \\
Logro Esperado & 2 & $9.5 \%$ & 11 & $52.4 \%$ & 9 & $42.9 \%$ \\
Logro Destacado & 0 & $0.0 \%$ & 7 & $33.3 \%$ & 7 & $33.3 \%$ \\
Total & 21 & $100.0 \%$ & 21 & $100.0 \%$ & &
\end{tabular}

Fuente: Base de datos del pre y post test.

Figura 2 Frecuencia del equilibrio ecológico antes y después de aplicar las estrategias de reciclaje en niños, docentes y padres de familia del CEBE Fe y Alegría $N^{\circ}$ 42, 2020-2021.

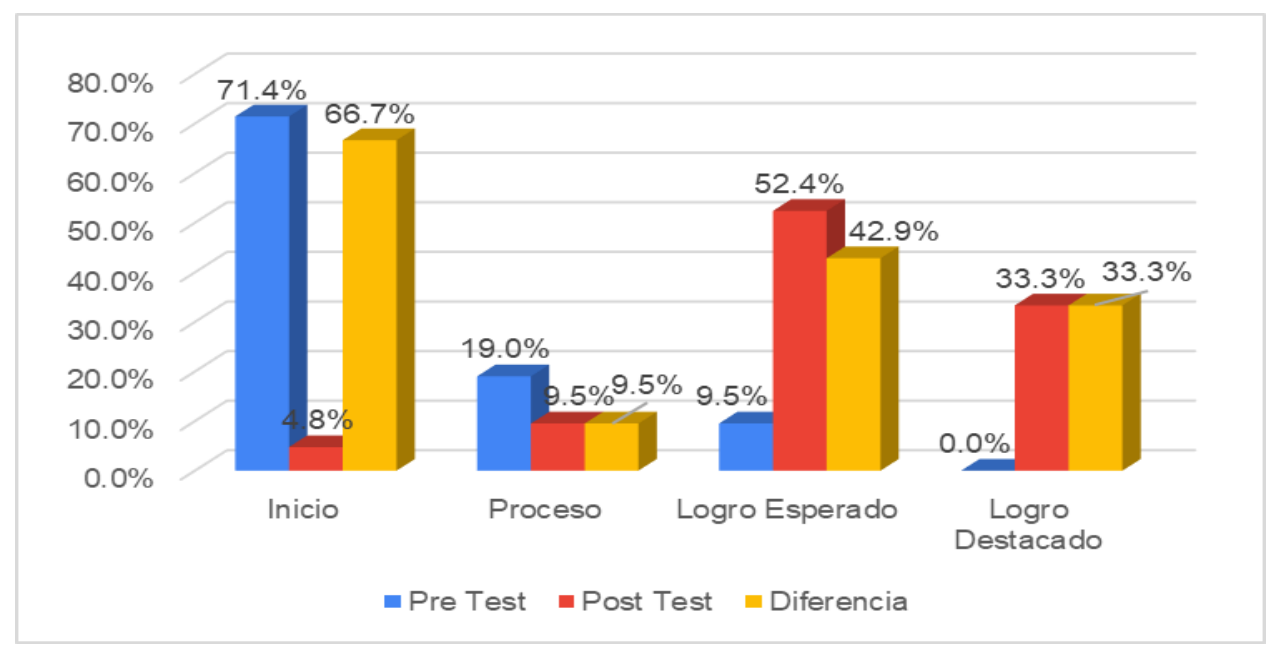

Fuente: Tabla 2

\section{Interpretación:}

A partir de los resultados de la comparación realizada en el pre y post test se tiene que en el nivel de inicio en el pre test se ha evidencia a 15 estudiantes que representan el $71.4 \%$, luego en el post test se ha registrado a 1 estudiante que conforma el $4.8 \%$, logrando disminuir en $66.7 \%$; de ahí se tiene que en el nivel proceso en el pre test se ha evidenciado que 4 estudiantes que conforman el $19.0 \%$ y en el post test se ha mostrado a 2 estudiante que conforma el 9.5\%, logrando una mejora del 9.5\%. Luego en el nivel logro esperado 
se tiene en el pre test a 2 estudiantes que conforman el 9.5\%, luego en el post test se tiene que 11 estudiantes que conforman el 52.4\%, haciendo una diferencia del $42.9 \%$. Finalmente, en el nivel logro destacado en el pre test no se tiene registro y en el post test se ha registrado a 7 estudiantes que conforman el 33.3\%. haciendo una diferencia del $33.7 \%$, demostrando la efectividad que presento las estrategias de reciclaje.

Tabla 3 Comparar los resultados de los valores ambientales antes y después de aplicar las estrategias de reciclaje en niños, docentes y padres de familia del CEBE Fe y Alegría $N^{\circ} 42,2020-2021$.

\begin{tabular}{lcccccc}
\hline \multirow{2}{*}{ Niveles } & \multicolumn{2}{c}{ Pre Test } & \multicolumn{2}{c}{ Post Test } & \multicolumn{2}{c}{ Diferencia } \\
& $\mathbf{f i}$ & $\mathbf{\%}$ & $\mathbf{f i}$ & $\mathbf{\%}$ & $\mathbf{f i}$ & $\mathbf{\%}$ \\
\hline Inicio & 8 & $38.1 \%$ & 0 & $0.0 \%$ & 8 & $38.1 \%$ \\
Proceso & 7 & $33.3 \%$ & 5 & $23.8 \%$ & 2 & $9.5 \%$ \\
Logro Esperado & 5 & $23.8 \%$ & 6 & $28.6 \%$ & 1 & $4.8 \%$ \\
Logro Destacado & 1 & $4.8 \%$ & 10 & $47.6 \%$ & 9 & $42.9 \%$ \\
Total & 21 & $100.0 \%$ & 21 & $100.0 \%$ & & \\
\hline
\end{tabular}

Fuente: Base de datos del pre y post test.

Figura 3 Frecuencia de los valores ambientales antes y después de aplicar las estrategias de reciclaje en niños, docentes y padres de familia del CEBE Fe y Alegría $N^{\circ}$ 42, 2020 - 2021.

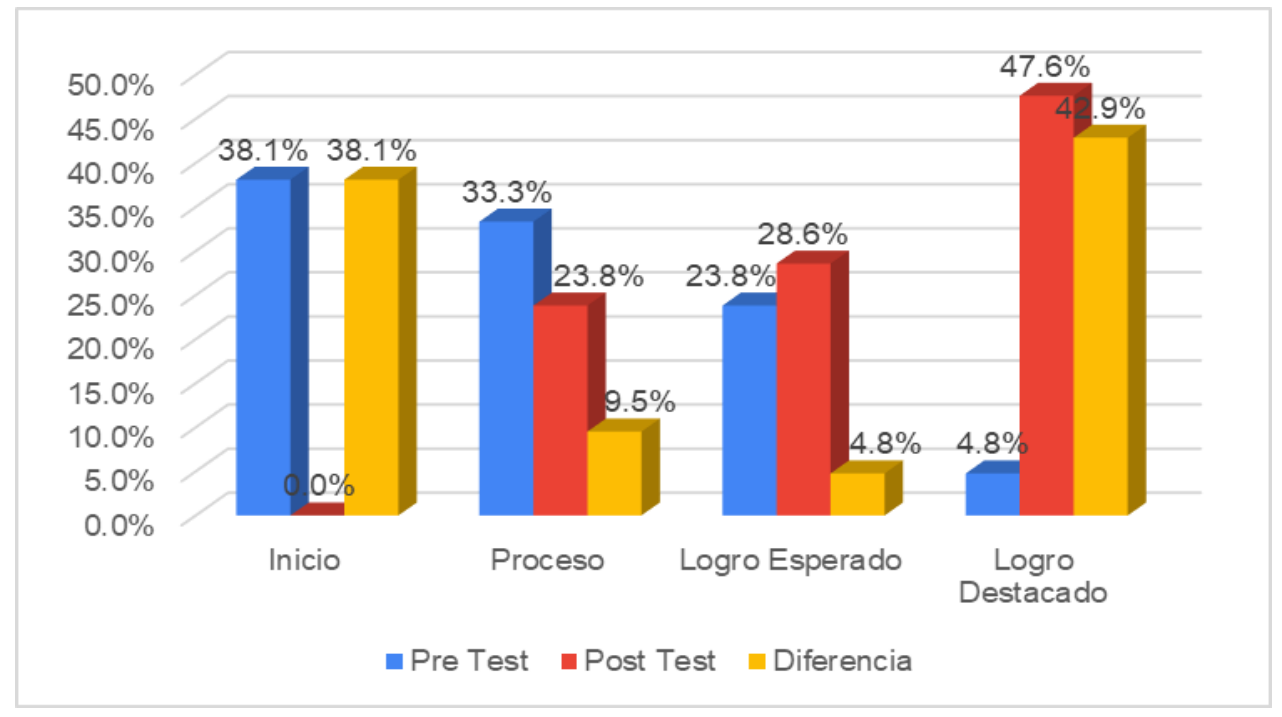

Fuente: Tabla 3

\section{Interpretación:}

A partir de los resultados de la comparación realizada en el pre y post test se tiene que en el nivel de inicio en el pre test se ha evidencia a 8 estudiantes que representan el $38.1 \%$, luego en el post test se ha registrado a 0 estudiante que conforma el $0.0 \%$, logrando disminuir en $38.1 \%$; de ahí se tiene que en el nivel proceso en el pre test se ha evidenciado 
que 7 estudiantes que conforman el $33.3 \%$ y en el post test se ha mostrado a 5 estudiante que conforma el 23.8\%, logrando una mejora del 9.5\%. Luego en el nivel logro esperado se tiene en el pre test a 5 estudiantes que conforman el $23.8 \%$, luego en el post test se tiene que 6 estudiantes que conforman el $28.6 \%$, haciendo una diferencia del $4.8 \%$. Finalmente, en el nivel logro destacado en el pre test se tiene 1 estudiante que conforman el $4.8 \%$ y en el post test se ha registrado a 10 estudiantes que conforman el $47.6 \%$. haciendo una diferencia del $42.9 \%$, demostrando la efectividad que presento las estrategias de reciclaje.

Tabla 4 Comparar los resultados del consumo responsable antes y después de aplicar las estrategias de reciclaje en niños, docentes y padres de familia del CEBE Fe y Alegría $N^{\circ} 42,2020-2021$.

\begin{tabular}{lcccccc}
\hline \multirow{2}{*}{ Niveles } & \multicolumn{2}{c}{ Pre Test } & \multicolumn{2}{c}{ Post Test } & \multicolumn{2}{c}{ Diferencia } \\
& $\mathbf{f i}$ & $\mathbf{\%}$ & $\mathbf{f i}$ & $\mathbf{\%}$ & $\mathbf{f i}$ & $\mathbf{\%}$ \\
\hline Inicio & 7 & $33.3 \%$ & 0 & $0.0 \%$ & 7 & $33.3 \%$ \\
Proceso & 2 & $9.5 \%$ & 1 & $4.8 \%$ & 1 & $4.8 \%$ \\
Logro Esperado & 12 & $57.1 \%$ & 9 & $42.9 \%$ & 3 & $14.3 \%$ \\
Logro Destacado & 0 & $0.0 \%$ & 11 & $52.4 \%$ & 11 & $52.4 \%$ \\
Total & 21 & $100.0 \%$ & 21 & $100.0 \%$ & & \\
\hline
\end{tabular}

Fuente: Base de datos del pre y post test.

Figura 4 Frecuencia del consumo responsable antes y después de aplicar las estrategias de reciclaje en niños, docentes y padres de familia del CEBE Fe y Alegría $N^{\circ}$ 42, 2020 - 2021.

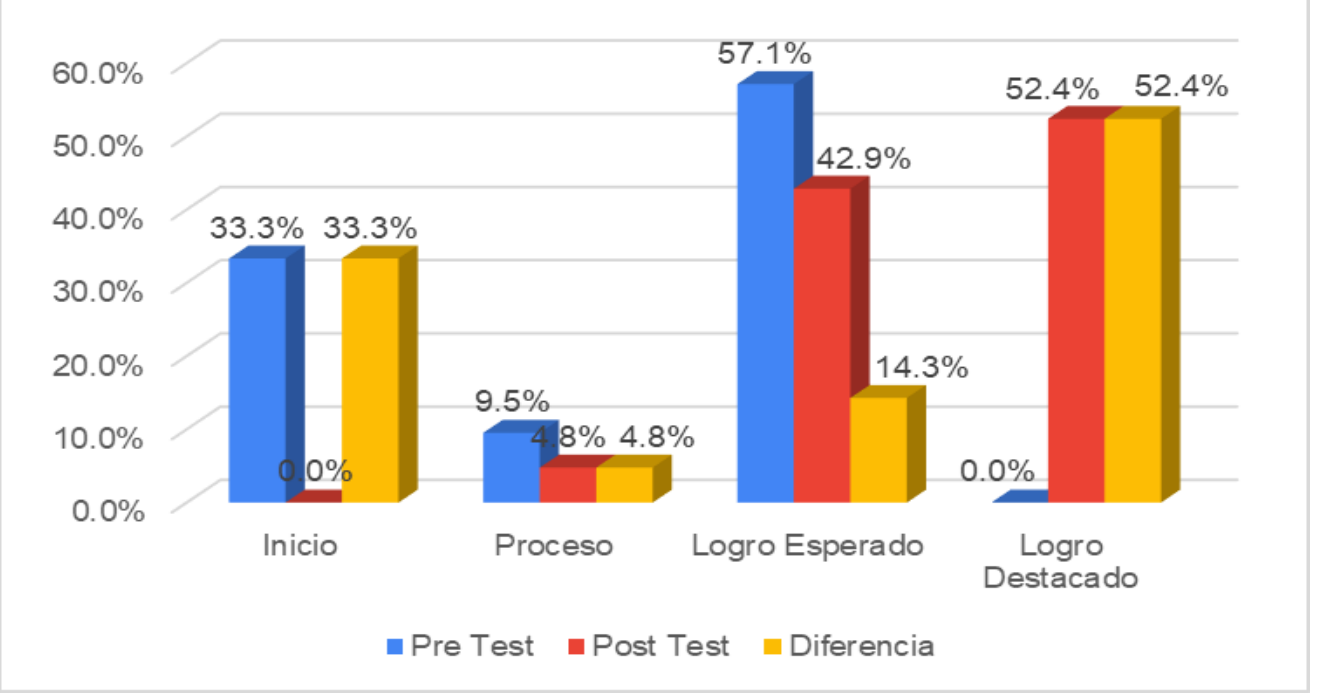

Fuente Tabla 4

\section{Interpretación:}

A partir de los resultados de la comparación realizada en el pre y post test se tiene que en el nivel de inicio en el pre test se ha evidencia a 7 estudiantes que representan el $33.3 \%$, 
luego en el post test se ha registrado a 0 estudiante que conforma el $0.0 \%$, logrando disminuir en $33.3 \%$; de ahí se tiene que en el nivel proceso en el pre test se ha evidenciado que 2 estudiantes que conforman el $9.5 \%$ y en el post test se ha mostrado a 1 estudiante que conforma el 4.8\%, logrando una mejora del $4.8 \%$. Luego en el nivel logro esperado se tiene en el pre test a 12 estudiantes que conforman el $57.1 \%$, luego en el post test se tiene que 9 estudiantes que conforman el $42.9 \%$, haciendo una diferencia del $14.3 \%$. Finalmente, en el nivel logro destacado en el pre test se tiene 0 estudiante que conforman el $0.0 \%$ y en el post test se ha registrado a 11 estudiantes que conforman el $52.4 \%$. haciendo una diferencia del 52.4\%, demostrando la efectividad que presento las estrategias de reciclaje.

Tabla 5 Comparar los resultados de la calidad de vida antes y después de aplicar las estrategias de reciclaje en niños, docentes y padres de familia del CEBE Fe y Alegría $N^{\circ}$ $42,2020-2021$.

\begin{tabular}{lcccccc}
\hline \multirow{2}{*}{ Niveles } & \multicolumn{2}{c}{ Pre Test } & \multicolumn{2}{c}{ Post Test } & \multicolumn{2}{c}{ Diferencia } \\
& $\mathbf{f i}$ & $\mathbf{\%}$ & $\mathbf{f i}$ & $\mathbf{\%}$ & $\mathbf{f i}$ & $\mathbf{\%}$ \\
\hline Inicio & 10 & $47.6 \%$ & 1 & $4.8 \%$ & 9 & $42.9 \%$ \\
Proceso & 5 & $23.8 \%$ & 2 & $9.5 \%$ & 3 & $14.3 \%$ \\
Logro Esperado & 4 & $19.0 \%$ & 6 & $28.6 \%$ & 2 & $9.5 \%$ \\
Logro Destacado & 2 & $9.5 \%$ & 12 & $57.1 \%$ & 10 & $47.6 \%$ \\
Total & 21 & $100.0 \%$ & 21 & $100.0 \%$ & & \\
\hline
\end{tabular}

Fuente: Base de datos del pre y post test.

Figura 5 Frecuencia de la calidad de vida antes y después de aplicar las estrategias de reciclaje en niños, docentes y padres de familia del CEBE Fe y Alegría $N^{\circ}$ 42, 2020 - 2021.

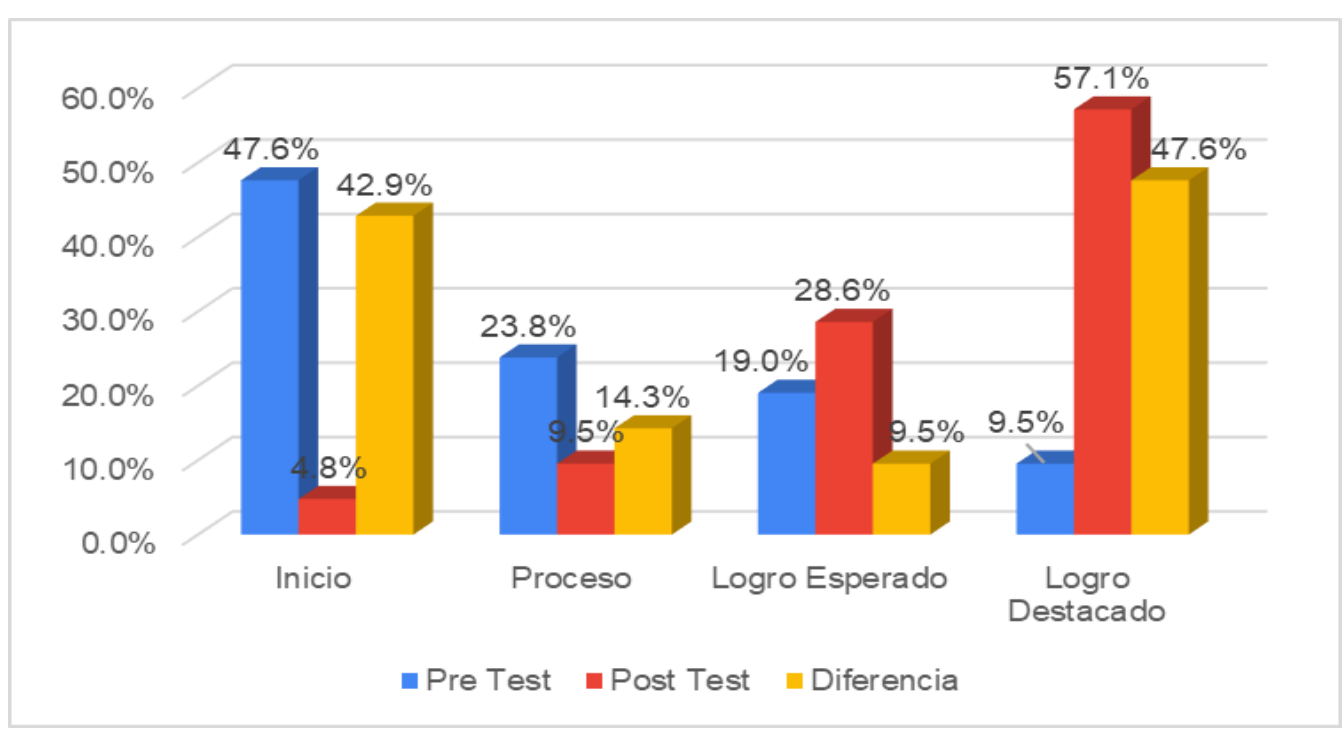

Fuente: Tabla 5 


\section{Interpretación:}

A partir de los resultados de la comparación realizada en el pre y post test se tiene que en el nivel de inicio en el pre test se ha evidencia a 10 estudiantes que representan el $47.6 \%$, luego en el post test se ha registrado a 1 estudiante que conforma el $4.8 \%$, logrando disminuir en $42.9 \%$; de ahí se tiene que en el nivel proceso en el pre test se ha evidenciado que 5 estudiantes que conforman el $23.8 \%$ y en el post test se ha mostrado a 2 estudiante que conforma el 9.5\%, logrando una mejora del 14.3\%. Luego en el nivel logro esperado se tiene en el pre test a 4 estudiantes que conforman el 19.0\%, luego en el post test se tiene que 6 estudiantes que conforman el $28.6 \%$, haciendo una diferencia del $9.5 \%$. Finalmente, en el nivel logro destacado en el pre test se tiene 2 estudiante que conforman el $9.5 \%$ y en el post test se ha registrado a 12 estudiantes que conforman el $57.1 \%$. haciendo una diferencia del $47.6 \%$, demostrando la efectividad que presento las estrategias de reciclaje.

\section{Prueba de hipótesis}

H0: La implementación de estrategias de reciclaje influye significativamente en el desarrollo la cultura ambiental de los niños, docentes y padres de familia del CEBE Fe y Alegría N 42, 2020 - 2021.

Ha: La implementación de estrategias de reciclaje no influye significativamente en el desarrollo la cultura ambiental de los niños, docentes y padres de familia del CEBE Fe y Alegría N 42, 2020 - 2021.

Nivel de significancia: $\alpha=0.05$

Estadístico de prueba: T de Student

\section{Tabla 6}

Prueba de hipótesis

\begin{tabular}{lccccc}
\hline Variable & \multicolumn{3}{c}{ Prueba T - Student } & Valor & Valor \\
Observado & tabular & $\begin{array}{c}\text { Probabilidad } \\
\text { significancia }\end{array}$ & $\begin{array}{c}\text { Nivel de } \\
\text { significancia }\end{array}$ & $\begin{array}{c}\text { to >tc } \\
\mathbf{p}<\alpha\end{array}$ \\
\hline Cultura & to $=11,040$ & tc $=1,725$ & $\mathrm{p}=0,0000$ & $\alpha=0,05$ & Se rechaza \\
ambiental & & & & $H_{0}$ \\
\hline
\end{tabular}

Fuente: Base de datos. 
Figura 6. Campana de Gauss

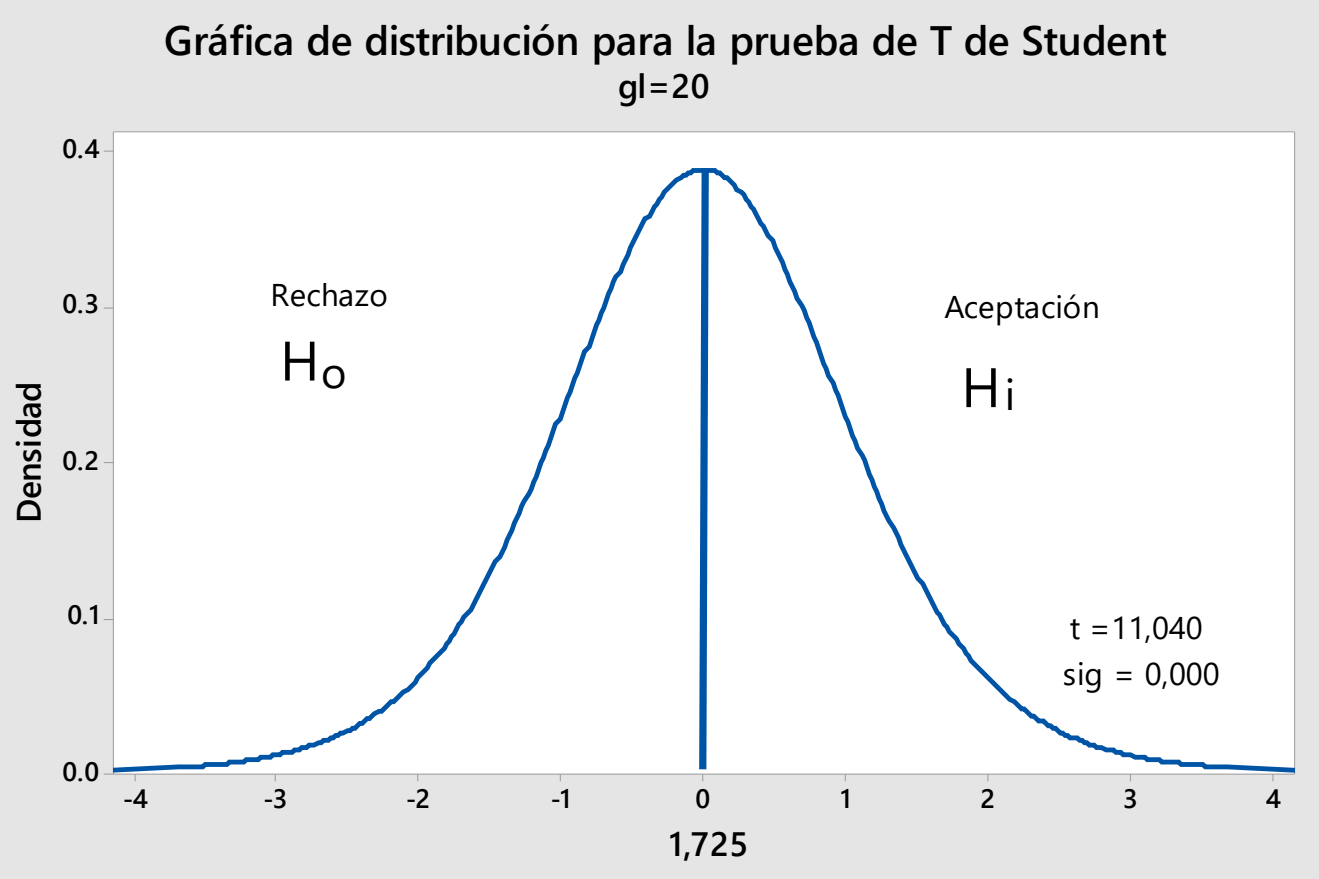

\section{Interpretación:}

Al realizar el análisis de la prueba de hipótesis se tiene la prueba T de Student para ello se ha logrado obtener un valor T observado de 11,040 considerado como superior al valor T tabular 1,725 (20 grados de libertad), con lo cual se considera que existe incidencia de las estrategias de reciclaje sobre el desarrollo la cultura ambiental, además al analizar el valor de la significancia obtenida se tiene un valor de 0.000 , situado por debajo del 0.05 con lo cual se procede al rechazo de la hipótesis nula y se comprueba la hipótesis de estudio afirmando que la implementación de estrategias de reciclaje influye significativamente en el desarrollo la cultura ambiental de los niños, docentes y padres de familia del CEBE Fe y Alegría N 42, 2020 - 2021.

\subsection{Discusión}

A partir de los resultados de la comparación realizada en el pre y post test al objetivo general que fue implementar estrategias de reciclaje para el desarrollo la cultura ambiental de los niños, docentes y padres de familia del CEBE Fe y Alegría $\mathrm{N}^{\circ} 42,2021$; se obtuvo que, en el nivel de inicio en el pre test se ha evidencia a 6 estudiantes que representan el $28.6 \%$, luego en el post test se ha registrado a ningún estudiante, logrando disminuir en $28.6 \%$; de ahí se tiene que en el nivel proceso en el pre test se ha evidenciado que 11 
estudiantes que conforman el $52.4 \%$ y en el post test se ha mostrado a 1 estudiante que conforma el $4.8 \%$, logrando una mejora del $47.6 \%$. Luego en el nivel logro esperado se tiene en el pre test a 4 estudiantes que conforman el $19.0 \%$, luego en el post test se tiene que 6 estudiantes que conforman el 28.6\%, haciendo una diferencia del 9.5\%. Finalmente, en el nivel logro destacado en el pre test no se tiene registro y en el post test se ha registrado a 14 estudiantes que conforman el $66.7 \%$. haciendo una diferencia del $66.7 \%$, demostrando la efectividad que presento las estrategias de reciclaje.

De acuerdo con los resultados obtenidos, se consideró a Díaz y Fuentes (2018) que realizaron un estudio acerca de las percepciones y significados de la concientización ambiental en estudiantes de primaria, para lograrlo se utilizó un método interaccional y simbólico, partiendo de una perspectiva hermenéutica-histórica y un modelo de investigación naturalista e interpretativa, las derivaciones correspondientes al estudio brindan saberes de cuatro dimensiones que favorecen el entendimiento del desarrollo de la concientización ambiental, lo que funda las bases para implementar programas educativos y elección de estrategias didácticas adecuadas que beneficien la concientización desde edades menores contribuyendo a la instrucción sostenible y conocedora de las problemáticas actuales. Por otro lado, para Arroyo (2016) en su estudio acerca de fomentar la cultura de reciclaje en Quito mediante un plan de comunicación, realizado dentro de un proyecto de inclusión del reciclaje.

En este estudio el autor logró concluir que utilizar planes estratégicos basados en la comunicación para fomentar la cultura de reciclaje en los habitantes de Quito resulto en la implementación de técnicas de producción efectivas que enseñaron a segregar adecuadamente los desechos según la clasificación de colores de los depósitos logrando interiorizar en ellos la cultura del reciclaje que será llevada hacia más pobladores de su entorno manteniendo una comunidad y ambiente saludables.

Asimismo, Obispo (2017) en su investigación acerca de la creación de un huerto escolar para la concientización ambiental de estudiantes de Villa El Salvador, tuvo como finalidad establecer la consecuencia de implementar un huerto escolar en el desarrollo de conciencia medioambiental en estudiantes de secundaria, la población estudiada se conformó por 220 educandos de los cuales se consideraron 70 para la muestra a los que se les evaluó mediante una encuesta en la que se utilizó la escala de Likert para establecer la medición de la variable además de un pre y post test. La variable estudiada se estructuró 
en cuatro dimensiones basadas en lo propuesto por Chuliá (1995), se propusieron 30 ítems que hicieron posible medir el impacto de la implementación del huerto escolar en la generación de conciencia hacia el medio ambiente. El tipo de investigación fue aplicada, de esquema cuasiexperimental, con un muestro no probabilístico e intencionado. La información se procesó mediante estadística descriptiva utilizando el coeficiente U de Mann Whitney para medir la significancia concluyéndose que el grupo experimental mejoró significativamente respecto del grupo control.

A partir de los resultados de la comparación realizada en el pre y post test se tiene que en el nivel de inicio en el pre test se ha evidencia a 15 estudiantes que representan el $71.4 \%$, luego en el post test se ha registrado a 1 estudiante que conforma el $4.8 \%$, logrando disminuir en $66.7 \%$; de ahí se tiene que en el nivel proceso en el pre test se ha evidenciado que 4 estudiantes que conforman el $19.0 \%$ y en el post test se ha mostrado a 2 estudiante que conforma el 9.5\%, logrando una mejora del 9.5\%. Luego en el nivel logro esperado se tiene en el pre test a 2 estudiantes que conforman el 9.5\%, luego en el post test se tiene que 11 estudiantes que conforman el 52.4\%, haciendo una diferencia del $42.9 \%$. Finalmente, en el nivel logro destacado en el pre test no se tiene registro y en el post test se ha registrado a 7 estudiantes que conforman el $33.3 \%$. haciendo una diferencia del $33.7 \%$, demostrando la efectividad que presento las estrategias de reciclaje.

Dados los resultados obtenidos, según Obispo (2017) en su investigación acerca de la creación de un huerto escolar para la concientización ambiental de estudiantes de Villa El Salvador, tuvo como finalidad establecer la consecuencia de implementar un huerto escolar en el desarrollo de conciencia medioambiental en estudiantes de secundaria, la población estudiada se conformó por 220 educandos de los cuales se consideraron 70 para la muestra a los que se les evaluó mediante una encuesta en la que se utilizó la escala de Likert para establecer la medición de la variable además de un pre y post test.

La variable estudiada se estructuró en cuatro dimensiones basadas en lo propuesto por Chuliá (1995), se propusieron 30 ítems que hicieron posible medir el impacto de la implementación del huerto escolar en la generación de conciencia hacia el medio ambiente. El tipo de investigación fue aplicada, de esquema cuasiexperimental, con un muestro no probabilístico e intencionado. La información se procesó mediante estadística descriptiva utilizando el coeficiente U de Mann Whitney para medir la significancia 
concluyéndose que el grupo experimental mejoró significativamente respecto del grupo control.

A partir de los resultados de la comparación realizada en el pre y post test se tiene que en el nivel de inicio en el pre test se ha evidencia a 8 estudiantes que representan el $38.1 \%$, luego en el post test se ha registrado a 0 estudiante que conforma el $0.0 \%$, logrando disminuir en $38.1 \%$; de ahí se tiene que en el nivel proceso en el pre test se ha evidenciado que 7 estudiantes que conforman el $33.3 \%$ y en el post test se ha mostrado a 5 estudiante que conforma el $23.8 \%$, logrando una mejora del 9.5\%. Luego en el nivel logro esperado se tiene en el pre test a 5 estudiantes que conforman el $23.8 \%$, luego en el post test se tiene que 6 estudiantes que conforman el 28.6\%, haciendo una diferencia del $4.8 \%$. Finalmente, en el nivel logro destacado en el pre test se tiene 1 estudiante que conforman el $4.8 \%$ y en el post test se ha registrado a 10 estudiantes que conforman el $47.6 \%$. haciendo una diferencia del $42.9 \%$, demostrando la efectividad que presento las estrategias de reciclaje.

De acuerdo a los resultados obtenidos, se consideró a Escobar (2018) quien, realizó un programa de formación ambientalista para desarrollar hábitos en estudiantes de San Juan de Miraflores, en el que se empleó una metodología aplicada y un esquema cuasiexperimental con el propósito de determinar el impacto del programa mencionado en los sujetos que participen de él, se tuvieron dos grupos, uno experimental y otro de control. A través de este estudio se concluyó en rechazar la hipótesis nula puesto que el valor obtenido fue menor a 0.025 , aceptándose así la hipótesis alternativa que sostiene que a través de la aplicación del programa hubo una mejora en las prácticas de los estudiantes integrantes del grupo experimental hacia el medio ambiente, lo que además se demostró mediante los talleres realizados y evaluados con listas de cotejo y guías de observación.

Por su parte, Correa y Arteaga (2013) que ejecutaron la implementación de un plan comunicacional estratégico como parte de un proyecto de reciclaje en Cartagena de Indias con el propósito de optar al título de Comunicadores sociales, concluyeron que el diseño del plan de comunicación estratégico evidenció que los habitantes de esa zona no tienen conocimientos sobre el reciclaje, sin embargo, se encuentran dispuestos a recibir la información y capacitación necesaria para cuidar su ambiente mediante el reciclaje. De igual forma hicieron notar su incomodidad respecto a la nula diferenciación de los 
contenedores de basura puesto que no se practica la segregación de los desechos de forma correcta.

A partir de los resultados de la comparación realizada en el pre y post test se tiene que en el nivel de inicio en el pre test se ha evidencia a 7 estudiantes que representan el $33.3 \%$, luego en el post test se ha registrado a 0 estudiante que conforma el $0.0 \%$, logrando disminuir en $33.3 \%$; de ahí se tiene que en el nivel proceso en el pre test se ha evidenciado que 2 estudiantes que conforman el $9.5 \%$ y en el post test se ha mostrado a 1 estudiante que conforma el $4.8 \%$, logrando una mejora del $4.8 \%$. Luego en el nivel logro esperado se tiene en el pre test a 12 estudiantes que conforman el $57.1 \%$, luego en el post test se tiene que 9 estudiantes que conforman el $42.9 \%$, haciendo una diferencia del $14.3 \%$. Finalmente, en el nivel logro destacado en el pre test se tiene 0 estudiante que conforman el $0.0 \%$ y en el post test se ha registrado a 11 estudiantes que conforman el $52.4 \%$. haciendo una diferencia del $52.4 \%$, demostrando la efectividad que presento las estrategias de reciclaje.

Al analizar los resultados obtenidos se consideró a Salazar (2017) quien realizó un programa para tratar los desechos sólidos basado en las 3R con el fin de mejorar las conductas ambientales en la Escuela Perfeccionamiento Docente (EPD) de Cajamarca, trabajando con una población total de 88 estudiantes de la cual se seleccionó una muestra de 23, previamente a la aplicación del programa se realizó un pre test en el que más del $70 \%$ de los encuestados se mostró indiferente hacia las problemáticas ambientales, tras la aplicación del programa a través de talleres y charlas de concientización se obtuvieron los siguientes resultados en el post test con un $74 \%$ de encuestados manifestándose muy de acuerdo en la implementación de estrategias que permitan y posibiliten el cuidado del medio ambiente, sumado a ello se evidencio que ningún participante se sentía indiferente ante la situación.

Por su parte Almeida (2016) realizó una indagación enfocada en establecer el grado de conciencia ambiental presente en los estudiantes de primaria de una institución educativa de Los Olivos, para ello se empeló una metodología de tipo básica, con un esquema descriptivo-no experimental y perspectiva cuantitativa, con la finalidad de realizar la medición se utilizó un cuestionario validado por expertos y aplicado a un total de150 individuos. Posterior al análisis y procesamiento de los datos se tiene que los educandos evaluados presentan un nivel intermedio de conciencia sobre el medio ambiente en un 
$75 \%$ mientras que el $25 \%$ restante presentan un nivel bajo, por lo que se hace evidente que la problemática ambiental debe ser un tópico reforzado y generalizado en las escuelas. Los autores Quiroga y Talledo (2016) por su parte, ejecutaron una estrategia basada en videos educativos para potenciar la conciencia ambiental de los educandos de una escuela secundaria en Trujillo con el propósito de optar por el título de licenciados en Ciencias de la Comunicación. Mediante su estudio concluyeron que los videos educativos o pedagógicos son una herramienta beneficiosa para los estudiantes puesto que, al ser medios audiovisuales, llevan de manera más rápida y didáctica la información obteniendo mejor disposición de los discentes que lograron interiorizar el mensaje y adquirieron una creciente preocupación por el cuidado de la naturaleza.

A partir de los resultados de la comparación realizada en el pre y post test se tiene que en el nivel de inicio en el pre test se ha evidencia a 10 estudiantes que representan el $47.6 \%$, luego en el post test se ha registrado a 1 estudiante que conforma el $4.8 \%$, logrando disminuir en $42.9 \%$; de ahí se tiene que en el nivel proceso en el pre test se ha evidenciado que 5 estudiantes que conforman el $23.8 \%$ y en el post test se ha mostrado a 2 estudiante que conforma el $9.5 \%$, logrando una mejora del 14.3\%. Luego en el nivel logro esperado se tiene en el pre test a 4 estudiantes que conforman el $19.0 \%$, luego en el post test se tiene que 6 estudiantes que conforman el 28.6\%, haciendo una diferencia del $9.5 \%$. Finalmente, en el nivel logro destacado en el pre test se tiene 2 estudiante que conforman el $9.5 \%$ y en el post test se ha registrado a 12 estudiantes que conforman el $57.1 \%$. haciendo una diferencia del $47.6 \%$, demostrando la efectividad que presento las estrategias de reciclaje.

Los resultados que se han obtenido fueron contrastados con Huamán, E. (2016) en su estudio acerca de los valores y la conciencia ambiental en los educandos de los Olivos, contaron con una porción de población conformada por 220 individuos seleccionados por muestro aleatorio simple los cuales se evaluaron mediante una escala de conducta ecológica y un cuestionario de valores propuesto por Casas, ambos instrumentos obtuvieron su validez por juicio de expertos con un índice de confianza mayor a 0.8 , concluyéndose que la concientización ambiental tiene una correspondencia elevada con los valores presentes en los educandos, según el valor de Rho de Spearman Rho = 7.33, asimismo, con este resultado quedó demostrada la hipótesis general. 
Benites y Solano (2016) en su investigación en la que implementaron un programa de reciclaje relacionado con el área de matemática para desarrollar además de las competencias del parea, saberes y conductas ambientales pertinentes en estudiantes de inicial, lograron concluir que a nivel del área de matemática, los niños progresaron exitosamente en sus capacidades favoreciendo su pensamiento lógico, de seriación y clasificación además de ello, al trabajar con materiales reciclados a modo de estrategia didáctica se le permitió a los estudiantes a muy temprana edad, acercarse a las nociones de reciclaje y aprovechamiento de residuos resultando beneficioso para su formación académica y moral.

También se consideró el estudio de Medina (2015) quien realizó un estudio sobre el mejoramiento de la conciencia ambiental de los discentes de una escuela ecológica en Arequipa partiendo de la implementación de proyectos ambientales, con el propósito de desarrollar conciencia medioambiental en dichos alumnos utilizando la técnica basada en proyectos, llegando a la conclusión siguiente: el grado de concientización ambiental de los educandos precio a la aplicación de los proyectos ambientales era mínimo, con una responsabilidad escasa con la conservación de la naturaleza, esto se puso de manifiesto en el resultado de 24 puntos de un total de 80 obtenido de la encuesta, mientras que tras realizar la aplicación de los proyectos este puntaje aumentó considerablemente resultando en 70 puntos de los 80 totales referidos en los instrumentos de evaluación, por lo que se hace notorio el impacto de los proyectos ecológicos en los estudiantes los cuales desarrollaron capacidades y competencias favorables y responsables con su medio ambiente.

\section{CONCLUSIONES}

Primera : Luego del análisis de los resultados se concluyó que, la implementación de estrategias de reciclaje influye significativamente en el desarrollo la cultura ambiental de los niños, docentes y padres de familia del CEBE Fe y Alegría $\mathrm{N}^{\circ} 42,2021$, con lo cual se procede al rechazo de la hipótesis nula y se comprueba la hipótesis de estudio, ya que, al realizar el análisis de la prueba de hipótesis se tiene la prueba T de Student, se ha logrado obtener un valor $\mathrm{T}$ observado de 11,040 considerado como superior al valor T tabular 1,725 (20 grados de libertad), el valor de la significancia obtenida se tiene un valor de 0.000 , situado por debajo del 0.05 . 
Segunda : Al analizar los resultados producto del análisis de los resultados obtenidos para el primer objetivo específico se concluyó que el equilibrio ecológico se desarrolló de manera destacada, puesto que, en el nivel logro destacado en el pre test no se tiene registro y en el post test se ha registrado a 14 estudiantes que conforman el $66.7 \%$. haciendo una diferencia del $66.7 \%$, demostrando la efectividad que presento las estrategias de reciclaje.

Tercera : Luego del análisis de los resultados obtenidos para el objetivo específico segundo en cuanto a los valores ambientales se concluyó que, se desarrolló en el nivel logro destacado, debido a que en el pre test se tiene 1 estudiante que conforman el $4.8 \%$ y en el post test se ha registrado a 10 estudiantes que conforman el $47.6 \%$. haciendo una diferencia del $42.9 \%$, demostrando la efectividad que presento las estrategias de reciclaje.

Cuarta : Después de analizar los resultados obtenidos en la investigación en lo referente al tercer objetivo específico sobre el consumo responsable, se concluyó que, se logró un nivel destacado evidenciado en el pre test ya que se tiene 0 estudiante que conforman el $0.0 \% \mathrm{y}$ en el post test se ha registrado a 11 estudiantes que conforman el 52.4\%. haciendo una diferencia del $52.4 \%$, demostrando la efectividad que presento las estrategias de reciclaje.

Quinta : Evidenciado en los resultados obtenidos para el objetivo específico cuarto en cuanto a calidad de vida, se concluyó que, se logró desarrollar el nivel logro destacado en los educandos dado que, en el pre test se tiene 2 estudiante que conforman el $9.5 \%$ y en el post test se ha registrado a 12 estudiantes que conforman el $57.1 \%$. haciendo una diferencia del $47.6 \%$, demostrando la efectividad que presento las estrategias de reciclaje.

\section{LISTA DE REFERENCIAS}

Arroyo, A. (2016). Plan de comunicación para incentivar la cultura del reciclaje en la ciudad de Quito, como parte del proyecto de la iniciativa para el reciclaje inclusivo (IRR). Quito. Universidad de las Américas. 
Almeida, K. (2016) Conciencia Ambiental en estudiantes de sexto grado de educación primaria de la Institución Educativa 2090 "Virgen de la Puerta"- Los Olivos2015. Recuperado de: https://repositorio.ucv.edu.pe/handle/20.500.12692/103

Beckmann, S.C. (2007). "Consumers and corporate social responsibility: Matching the unmatchable?", Australasian Marketing Journal, vol. 15, nº 1, págs. 27-36.

Benites, S. y Solano, T. (2016) Programa "Reciclaeduca" para el desarrollo de operaciones matemáticas de clasificación y seriación en niños y niñas de 4 años de la I.E 215, Urbanización Miraflores de la Ciudad de Trujillo-2014.

Carrigan, M. y Atalla, A. (2001). "The Myth of the Ethical Consumer, Do Ethics Matter in Purchase Behaviour?”, Journal of Consumer Marketing, vol. 18, nº 7, págs. 560-577.

Castells, E. (2012). Clasificación y gestión de residuos. Colección Monografías. España. Edit. Díaz de Santos.

Castells, E. (2012). Reciclaje de residuos industriales. 2a ed. España. Edit. Díaz de Santos.

Castillo, I. (11 de June de 2019). Cultura ambiental: concepto, importancia, ejemplos. Lifeder. Recuperado de https://www.lifeder.com/culturaambiental/.

Carrillo, A. (2015). Población y Muestra. Recuperado de: http://ri.uaemex.mx/oca/view/20.500.11799/35134/1/secme-21544.pdf.

Cassierra, L. (2015). Sistematización de la implementación de un Proyecto Educativo para el reciclaje de residuos sólidos y su reutilización en artesanías con estudiantes de la Institución Educativa Politécnico Municipal, sede "Célimo Rueda", Santiago de Cali, Valle del Cauca. Tesis de Maestría, Universidad de Manizales, Colombia. CMAP. (2013, septiembre 16). Environmental Benefits. Tomado de cmap.illinois.gov.

Commoner, B. (1971). The Closing Circle: Nature, Man and Technology. New York: Alfred Knopf.

Cooper, D. E. y Palmer, J.A. (1995). Just environments. Intergenerational, international and interspecies issues. London-New York: Routledge. 
Cooper, M. E. y Holbrook, M. B. (1993). "Ethical consumption experiences and ethical space”, Advances in Consumer Research, vol. 20, págs. 113-118. Cortina, A. (2003) Por una ética del consumo, Madrid: Taurus

Correa, C, \& Arteaga, P. (2013). Plan estratégico de comunicación Barrio Cero Basura fase I-Diseño de prueba piloto para proyecto de reciclaje "CONSERVARED” de la fundación Conservaré en el barrio de Getsemaní de Cartagena de Indias. Universidad de Cartagena, Cartagena.

Daly, H.E. (1999). Ecological Economics and the Ecology of Economics: Essays in Criticism. Cheltenham: Edward Elgar Pub.

De Los Ríos, G. (2018) Aplicación del plan nacional de educación ambiental en el desarrollo de la conciencia ambiental de los estudiantes de primaria en las escuelas ecoeficientes del distrito de San Juan de Lurigancho UGEL 05. Lima. UCV.

Díaz, J. y Fuentes, F. (2018) desarrollo de la conciencia ambiental en niños de sexto grado de educación primaria. significados y percepciones. CPU-e. Rev. Investig. Educ No.26 Xalapa ene./jun. 2018. Recuperado de: http://www.scielo.org.mx/scielo.php?script=sci_arttext\&pid=S18705308201800 0100136.

Diaz y otros, (Publicado el 13 de junio del 2016) Diseño de un sistema de segregación y educación sobre el reciclaje para los colegios Montessori y Turicará. Universidad de Piura. Escuela de Posgrado. Facultad de Ingeniería.

Environmental education. (s/f). Defining Environmental Education. Tomado de gdrc.org

Escobar, A. Quintero, D. y Serradas D. (2006). El Reciclaje como instrumento para la Concientización de la Conservación del Ambiente. Venezuela. Tesis inédita. Universidad Andrés Bello.

Escobar, J. (2018) Programa de Educación Ambiental para mejorar hábitos en niños de la I.E. Naciones Unidas de San Juan de Miraflores, 2018.

Hino Motors. (s/f). Environmental Education. Tomado de hino-global.com. Huamán, E. (2016) Conciencia ambiental y los valores en los estudiantes de 6to grado de primaria en la Red educativa $N^{o}$ 17. Los Olivos, 2016. Recuperado de: https://repositorio.ucv.edu.pe/bitstream/handle/20.500.12692/4945/Huaman_M EM.pdf?sequence=1\&isAllowed=y 
López, M. (2012). Anuncian proyecto para reciclar basura. Prensa Libre. Reportaje:

López Pérez, M. D. (2017). Gestión de residuos inertes: UF0286. Recuperado de: http://ebookcentral.proquest.com/lib/bibliourpsp/detail.action?docID=5214014

Martínez, S y Bigues, J. (2009) El libro de las 3R. Contribuidores Proyecto Nature-libro, Corp. Editor Nuevos Emprendimientos, 2009.

Meadows, D.; Meadows, D.; Randers, J. (1992). Beyond the limits. Global collapse or a sustainable future. London, Earthscan; Más allá de los límites del crecimiento. Madrid: El País-Aguilar.

Medina, M. A. (2015). Implementación de metodologías para la gestión de residuos de construcción y demolición en edificaciones de vivienda de material noble en Lima. UCV.

Medina, K. (2015) Fortalecimiento de la conciencia ambiental de las estudiantes del V ciclo nivel de educación primaria de la Escuela ecológica urbana San Lorenzo 40020, mediante la realización de proyectos ecológicos Arequipa-2014. Recuperado de: http://repositorio.unsa.edu.pe/bitstream/handle/UNSA/1982/EDmellki.pdf

Ministerio del Ambiente (2016). MINAM "Crea conciencia en los niños sobre la contaminación sonora a través de actividades en el marco del DIAIRE”, 2016. Lima, agosto. Recuperado de http://www.minam.gob.pe/reeduca/2016/08/12/actividades-del-minampor-eldía-interamericano-de-la-calidad-del-aire-diaire/

Ministerio del Ambiente (2019). MINAM "La conciencia ambiental se define como el conocimiento, la internalización de valores y la solución de la problemática ambiental". Recuperado de http://www.minam.gob.pe/reeduca/2016/08/12/actividades-del-minampor-eldia-interamericano-de-la-calidad-del-aire-diaire/

Naess, A. [1973] (1989). Ecology, Community and Lifestyle. An outline of an ecosophy. Cambridge: Cambridge University Press.

Obispo, Y. (2018) "Programa huerto escolar en la conciencia ambiental en estudiantes de la Institución Educativa República de Bolivia, Villa El Salvador-2017”. Recuperado de: https://core.ac.uk/download/pdf/154580817.pdf

Pachamama. (s/f). Environmental Awareness. Tomado de pachamama.org. 
Parellada, R. (2012). Reciclaje, no se necesitan leyes que lo impulsen. Siglo XXI del 23 de agosto. Guatemala.

Pardavé, W. (2007). Estrategias ambientales de las 3R a las 10R. Colección Textos Universitarios, CEP. Colombia.

Pérez, L. (2007). Los Derechos de la Sustentabilidad. Argentina. Universidad Coliheu. Pepper, D. (1984). The roots of modern environmentalism. London: Croomhelm. Portocarrero, C., Zavaleta, N, Costilla, N. y Adrianzen, A. (2021). Educación ambiental y pensamiento crítico en la sociedad actual. Revista científica Yachaq, 4(1), 7791. doi: https://doi.org/10.46363/yachaq.v4i1.136

Quiroga, J, \& Talledo, G. (2016). El video educativo como estrategia de aprendizaje para mejorar la conciencia ambiental en los alumnos de secundaria de la I.E Pública San Martín de Porres N80036 del distrito de la Esperanza-Trujillo. Universidad Privada Antenor Orrego, Trujillo, Perú.

Raffino, M. (Última edición: 30 de septiembre de 2020) "Impacto ambiental". De: Argentina. Para: Concepto. de. "Impacto ambiental" Disponible en: https://concepto.de/impacto-ambiental/. Consultado: 11 de mayo de 2021.

Sainz de Vicuña, J. (2017). El plan estratégico en la práctica. Recuperado de: https://books.google.com.pe/books?hl=es\&lr=\&id=69YzDwAAQBAJ\&oi=f nd\&pgPA101\&dq=etapas+del+plan+estrategico\&ots=z2nsnutxBu\&sig=1uRnF Vd_B2qKyRcCfP2ouAwoI\#v=onepage\&q=etapas\%20del\%20plan\%20estrategi $\operatorname{co\& } \mathrm{f}=\mathrm{fals}$

Salazar, E. (2017) Programa de Manejo de Residuos sólidos basados en las 3R para mejorar las actitudes ambientales en la Escuela Perfeccionamiento Docente (EPD) de la Universidad Nacional de Cajamarca 2017. Recuperado de: https://repositorio.ucv.edu.pe/bitstream/handle/20.500.12692/25604/Salazar_CE F.pdf? sequence $=1 \&$ is Allowed $=\mathrm{y}$

Sistema Nacional de Gestión Ambiental (2016). SGNA. Cuidado del medio ambiente. Recuperado de http://www.minam.gob.pe/gestion-ambiental/quienessomos/

Schumacher, E.F. (1973). Small is beautiful: A study of economics as if People Mattered. New York: Harper y Row.

Schweitzer, A. (1946). Civilization and Ethics. London: A y C Black. 
Taylor, P. (1986). Respect for Nature: A theory of environmental ethics. Princeton: N.J. Princeton University Press

UNESCO. (s/f). La educación ambiental. Las grandes orientaciones de la Conferencia de Tbilisi. Tomado de unesdoc.unesco.org. Vargas, T. (2012). Basura Cero: Una alternativa sustentable.

Vela, R. (1990). Manned space stations. Their construction, operation and potential application. Paris. (1990)

Vincent, A. (1992). Modern political ideologies. Oxford: Blackwell.

Weston, A. (2004). What if teaching went wild. Canadian Journal of Environmental Education, Fondo de Cultura Económica.

Ward, B. y Dubos, R. (1972). Only One Earth. The care and maintenance of a small planet. New York, W.W. Norton 6 Co.; Una sola tierra. México: Fondo de Cultura Económica.

Yasvin, V.A. (2006). Building an Environmental Culture is not Reduced to Environmental Education. En Bulletin Towards a Sustainable Russia, Nro. 34, pp. 7-9.

Yurén, M.T. (1995). Eticidad, valores sociales y educación. México: Universidad Pedagógica Nacional. London: A y C Black. 